Pacific Journal of Mathematics

ON CANONICAL CONFORMAL MAPS OF REGIONS OF 


\title{
ON CANONICAL CONFORMAL MAPS OF REGIONS OF ARBITRARY CONNECTIVITY
}

\author{
EDGaR REICH AND S. E. WARSCHAWSKI
}

Introduction. The purpose of this paper is:

1. To present a simple proof for the existence of a conformal map of an arbitrary bounded region onto a circular slit disk and-if the region possesses at least one isolated boundary components which is not a point-onto a radial slit disk ${ }^{1}$

2. To remark on certain geometric properties and problems of uniqueness of the canonical slit domains thus obtained. The possibility of mapping regions of finite connectivity onto (circular and radial) slit disks was originally considered from the point of view of various variational problems by Koebe [8] and Rengel [14], Schiffer [18], Nehari [11]. Extension to regions of infinite connectivity were made by Grötzsch [3,5], Schiffer [17] and more recently, within the framework of certain extremal problems on Riemann surfaces by Sario [16], Jurchescu [7], and in the book on Riemann Surfaces by Ahlfors and Sario [1], Chapter III, $\S 16 \mathrm{D} .^{2}$

In the present paper we consider, in connection with the circular slit mapping, an extremal problem which is suggested by the now common proof of Riemann's mapping theorem..$^{3}$ Let $\Omega$ be a bounded region which contains the origin, and let $\mathscr{F}$ denote the family of functions $f(z)$ with the properties:

1. $f(z)$ is analytic and univalent in $\Omega$

2. $f(0)=0, f^{\prime}(0)>0,|f(z)| \leq 1$ in $\Omega$.

(Note that nothing regarding the boundary behavior of $f$ is predicated in the definition of $\mathscr{F}$.) We shall show that the function $\phi \in \mathscr{F}$ which solves the extremal problem $\phi^{\prime}(0)=\max _{f \in \mathscr{F}} f^{\prime}(0)$, gives a mapping of $\Omega$ onto a circular slit disk. The proof that $\phi(\Omega)$ is a circular slit disk may be based on a well known inequality of Rengel [13]. We derive it, however, from a more general lemma (Lemma 1) which enables us, in addition, to discuss further properties of $\phi$, in particular the uniqueness aspect of the solution. For a given $\Omega$ there may be more than one

Received September 30, 1959. The preparation of this paper was sponsored in part by the Office of Naval Research. Reproduction in whole or in part is permitted for any purpose of the United States Government.

1 A region is called a circular slit (radial slit) disk if a circle $|z|=r$ is one of the components of its boundary and if all other boundary components are circular arcs in $|z|<r$ concentric with $|z|=r$ (radial segments contained in $0<|z|<r$ ), or points other than the origin.

2 See also K. Oikawa's doctoral dissertation [12], Chapter III, in which he states that the circular slit disk mapping may be obtained from Sario [15].

3 Cf. also Nehari [11], p. 352 , for the case of finite connectivity. 
extremal function $\phi$, even in the case of finite connectivity. However, one achieves uniqueness of the solution of the extremal problem if one replaces the class $\mathscr{F}$ by the subclass $\mathscr{F}^{*}=\mathscr{F}^{*}(\Omega)$, whose elements $f$ are further restricted by the requirement that they carry the outer boundary component of $\Omega$ into $|w|=1$ ( $\S 4$ and 5 ).

To obtain an insight into all possible solutions $\phi$ of the extremal problem in $\mathscr{F}(\Omega)$ we consider the broader problem of the uniqueness of a properly normalized conformal map of $\Omega$ onto a circular slit unit disk. It is well known that the map $f$ of a bounded region $\Omega$, normalized by the condition that $f \in \mathscr{F}^{*}(\Omega)$, onto a circular slit unit disk $\Sigma$ is not necessarily unique, unless $\Sigma$ is further restricted. A characterization of such disks $\Sigma$ onto which there exists only one normalized conformal map was given by Grötzsch [5], p. 239 (Normalbereiche). In this paper we obtain a characterization of these disks-which we detote as "extremal circular slit disks" -in terms of the extremal length $\lambda_{q}\{\gamma\}$ of all cycles $\gamma \subset \Sigma$ surrounding $z=0$ and lying in an annulus $q<$ $|z|<1$ which contains all slits of $\Sigma$ (Theorem 5). An extension of our fundamental Lemma 1 to extremal circular slit disks (Theorem 6) permits us to discuss certain features of the extremal function $\phi \in \mathscr{F}(\Omega)$.

The problem of mapping onto radial slit disks is treated by considering the $\min _{f \in \mathscr{F}^{*}}\left|f^{\prime}(0)\right|$, where however $\Omega$ and $\mathscr{F}^{*}$ are subjected to an additional restriction. Similar results regarding the uniqueness of the extremal function and properties of the extremal domain are obtained here.

1. Mapping onto a circular slit disk. We begin with the principal lemma which is related to results of Grunsky [6] and Spencer [19].

LEMMA 1. Let $\Sigma$ be a circular slit unit disk centered at $z=0$ such that the circular projection $E$ of the boundary points of $\Sigma$ onto a fixed radius ${ }^{4}$ has linear measure zero. Suppose that $w=f(z)$ is analytic and univalent in $\Sigma$, that $f(0)=0$, and that $f(z)$ maps $\Sigma$ onto a bounded region $\Delta$ in such a manner that $|z|=1$ corresponds to the outer boundary component of $\Delta$. Let $F(z)=\log (f(z) / z), F(0)=\log f^{\prime}(0)(F(z)$ is singlevalued in $\Sigma$ once a branch of $\log f^{\prime}(0)$ is chosen). Let $A$ denote the area of 4 . Then

$$
\exp \left[\frac{1}{2 \pi} \iint_{\Sigma}\left|F^{\prime}(z)\right|^{2} d x d y\right]\left|f^{\prime}(0)\right| \leq \sqrt{\frac{A}{\pi}}
$$

In particular,

$$
\left|f^{\prime}(0)\right| \leq \sqrt{\frac{A}{\pi}}
$$

4 i.e. $E$ is the set of points on a fixed radius at which the circles $|z|=r$ containing a boundary point of $\Sigma$ intersect this radius. 
and equality implies $F^{\prime}(z) \equiv 0$, i.e. $f(z) \equiv a z$, where $a$ is a constant.

REMARKS. If $\Sigma$ has only a finite number of slits and if $|f(z)| \leq M$, (1') implies Rengel's inequality [13], $\left|f^{\prime}(0)\right| \leq M$, and the equality holds only if $f(z) \equiv a z$. If $\Sigma$ is the disk $|z|<1$, then $\left(1^{\prime}\right)$ becomes Bieberbach's area theorem.

Proof. It is easily seen that the set $E$ is closed. Consequently, given any $\varepsilon>0$, there exist a finite number of intervals, $\left[r_{\nu}, r_{\nu}^{\prime}\right]$ on $(0,1], r_{\nu}<r_{\nu}^{\prime}<r_{\nu+1}, \nu=1,2, \cdots, n-1$, such that $E \subset \bigcup_{\nu=1}^{n}\left[r_{\nu}, r_{\nu}^{\prime}\right]$ and that $\sum_{\nu=1}^{n}\left(r_{\nu}^{\prime}-r_{\nu}\right)<\varepsilon$. We may assume that no boundary points of $\Sigma$ are on $|z|=r_{\nu}$ and $|z|=r_{\nu}^{\prime}(\nu=1,2, \cdots, n-1)$ and on $|z|=r_{n}$, and that $r_{n}^{\prime}=1$.

Denote by $D_{\nu}$ the annulus $r_{\nu}^{\prime}<|z|<r_{\nu+1}(1 \leq \nu \leq n-1)$ and by $D_{0}$ the circle $|z|<r_{1} . \quad F(z)$ is single-valued and analytic in each $\bar{D}_{\nu}$. Assume first that $f^{\prime}(0)=1, F(0)=0$.

Let $z=r e^{i \theta}, F(z)=u+i v, f(z)=\rho e^{i \psi}$. Then ${ }^{5}$ in each $D_{\nu}, \nu=1,2, \cdots$, $n-1$,

$$
\iint_{D_{\nu}}\left|F^{\prime}(z)\right|^{2} d x d y=\int_{C_{\nu}} u d v=\int_{C_{\nu}} \log \frac{\rho}{r}(d \psi-d \theta)
$$

where $C_{\nu}$ is the boundary of $D_{\nu}$. Furthermore, we have for $D_{0}\left(C_{0}=\right.$ $\left\{|z|=r_{1}\right\}$ )

$$
\iint_{D_{0}}\left|F^{\prime}(z)\right|^{2} d x d y=\int_{c_{0}} \log \frac{\rho}{r}(d \psi-d \theta)
$$

Next we note that for $\nu=0,1, \cdots, n-1$

$$
\begin{aligned}
0 & =\mathfrak{\Im m}\left\{\int_{C_{\nu}} \frac{F(z)}{z} d z\right\}=\int_{c_{\nu}}\left[\log \frac{\rho}{r} d \theta+(\psi-\theta) \frac{d r}{r}\right. \\
& =\int_{C_{\nu}}[\log \rho d \theta-\log r d \psi r] .
\end{aligned}
$$

For $\nu=0$ this follows from the fact that $F(0)=0$, for $\nu>0$ this is a consequence of the regularity of $F(z) / z$ in $\bar{D}_{\nu}$. Thus we obtain

$$
\sum_{\nu=1}^{n-1} \int_{D_{\nu}} \int_{\nu}\left|F^{\prime}(z)\right|^{2} d x d y=\sum_{\nu=0}^{n-1} \int_{\sigma_{\nu}}\{\log \rho d \psi-2 \log r d \psi+\log r d \theta\} .
$$

We can evaluate the sum of the last two terms:

5 The following argument is similar to a technique used by Golusin [2], pp. 149-150. 
(2)

$$
\left\{\begin{array}{l}
\sum_{\nu=0}^{n-1} \int_{\sigma_{\nu}} \log r d \theta \\
=\sum_{\nu=1}^{n-1}\left\{\log r_{\nu} \int_{|z|=r_{\nu}} d \theta-\log r_{\nu}^{\prime} \int_{|z|=r_{\nu}^{\prime}} d \theta\right\}+\log r_{n} \int_{|z|=r_{n}} d \theta \\
=-2 \pi \sum_{\nu=1}^{n-1} \log \frac{r_{\nu}^{\prime}}{r_{\nu}}+2 \pi \log r_{n} .
\end{array}\right.
$$

The assumption that under the mapping $w=f(z)$, the circle $|z|=1$ corresponds to the outer component of $f(\Sigma)$, implies that the images of $|z|=r_{\nu}$ and $|z|=r_{\nu}^{\prime}$ are closed Jordan curves which contain $w=0$ in their interiors. Hence $\int_{|z|=r_{\nu}} d \psi=\int_{|z|=r_{\nu}^{\prime}} d \psi=2 \pi$. We obtain therefore in the same manner as above

$$
-\sum_{\nu=0}^{n-1} \int_{c_{\nu}} \log r d \psi=2 \pi \sum_{\nu=1}^{n-1} \log \frac{r_{\nu}^{\prime}}{r_{\nu}}-2 \pi \log r_{n} .
$$

Hence, combining (2) and (3) we find

$$
\begin{aligned}
\sum_{\nu=0}^{n-1}\left\{-2 \int_{c_{\nu}} \log r d \psi+\int_{c_{\nu}} \log r d \theta\right\} & =2 \pi \sum_{\nu=1}^{n-1} \log \frac{r_{\nu}^{\prime}}{r_{\nu}}-2 \pi \log r_{n} \\
& \leq 2 \pi \sum_{\nu=1}^{n-1} \frac{r_{\nu}^{\prime}-r_{\nu}}{r_{\nu}}+2 \pi \frac{1-r_{n}}{r_{n}} \\
& \leq \frac{2 \pi}{r_{1}} \sum_{\nu=1}^{n}\left(r_{\nu}^{\prime}-r_{\nu}\right)<\frac{2 \pi}{r_{1}} \varepsilon
\end{aligned}
$$

since $\sum_{\nu=1}^{n}\left(r_{\nu}^{\prime}-r_{\nu}\right)<\varepsilon$. Thus, if $\tau=\Sigma-\bigcup_{\nu=0}^{n-1} D_{\nu}$ and $|z| \leq d$ is a disk contained in $\Sigma$,

$$
\iint_{\Sigma}\left|F^{\prime}(z)\right|^{2} d x d y \leq \sum_{\nu=0}^{n-1} \int_{C_{\nu}} \log \rho d \psi+\frac{2 \pi}{d} \varepsilon+\iint_{Z}\left|F^{\prime}(z)\right|^{2} d x d y .
$$

Now, if $\Gamma_{\nu}=f\left(C_{\nu}\right), \Delta_{\nu}=f\left(D_{\nu}\right)$

$$
\int_{C_{\nu}} \log \rho d \psi=\int_{\Gamma_{\nu}} \log \rho d \psi=\iint_{\Delta_{\nu}} \frac{d u d v}{|w|^{2}}
$$

and if we remove a sufficiently small disk $k$ of radius $\mu$ about $w=0$,

$$
\int_{\sigma_{0}} \log \rho d \psi=\int_{\Gamma_{0}} \log \rho d \psi=\iint_{\Delta_{0}-k} \frac{d u d v}{|w|^{2}}+2 \pi \log \mu .
$$

Hence, if $f(\Sigma)=\Delta$,

$$
\sum_{\nu=0}^{n-1} \int_{o_{\nu}} \log \rho d \psi \leq \iint_{\triangle-k} \frac{d u d v}{|w|^{2}}+2 \pi \log \mu .
$$

Let $K=\{w|| w \mid<R\}$ be a disk with area $\pi R^{2}=A$. Then 


$$
\begin{aligned}
\iint_{-\kappa} \frac{d u d v}{|w|^{2}} & =\iint_{(\Delta-k) \cap K} \frac{d u d v}{|w|^{2}}+\iint_{\Delta-k-K} \frac{d u d v}{|w|^{2}} \leq \iint_{\mu \leq|w| \leq R} \frac{d u d v}{|w|^{2}} \\
& =2 \pi(\log R-\log \mu) .
\end{aligned}
$$

Thus we obtain from (4), (5) and (6)

$$
\iint_{\Sigma}\left|F^{\prime}(z)\right|^{2} d x d y \leq 2 \pi \log \sqrt{\frac{A}{\pi}}+\frac{2 \pi}{d} \varepsilon+\iint_{\tau}\left|F^{\prime}(z)\right|^{2} d x d y .
$$

Since the measure of $\tau$ is smaller than $2 \pi \varepsilon$ and the last inequality is true for every $\varepsilon>0$, we obtain the conclusion for the case $f^{\prime}(0)=1$. If $f^{\prime}(0) \neq 1$, we need only to replace $A$ by $A /\left|f^{\prime}(0)\right|^{2}$ and the lemma follows.

Let $\Omega$ be a bounded region, let $z=0 \in \Omega$, and let $\mathscr{F}$ denote the class of functions $f(z)$ with the properties:

1. $f(z)$ is analytic and univalent in $\Omega$

2. $f(0)=0, f^{\prime}(0)>0,|f(z)| \leq 1, z \in \Omega$.

Since $\mathscr{F}$ is normal there exists a function $\phi(z) \in \mathscr{F}$ such that $\phi^{\prime}(0) \geq$ $f^{\prime}(0)$ for any $f \in \mathscr{F}$ (note that $\phi^{\prime}(0)>0$ since $c z \in \mathscr{F}$ for some $c>0$, so that $\phi(z) \not \equiv$ constant $)$.

THEOREM 1. The function $w=\phi(z)$ maps $\Omega$ conformally onto a circular slit unit disk.

Proof. Let $\Omega^{\prime}=\phi(\Omega)$. Every point $w_{0},\left|w_{0}\right|=1$, is a boundary point of $\Omega^{\prime}$. If not, there exists a region $\Delta_{\varepsilon}$ in $|w|<1$ bounded by $|w|=1$ and an arc $\left\{\left|w-w_{0}\right|=\varepsilon,|w| \leq 1\right\}$, such that $\Omega^{\prime} \subset \Delta_{\varepsilon}$. If the function which maps $\Delta_{\varepsilon}$ onto $|w|<1$ and preserves the origin is composed with $\phi(z)$, then by the inequality (1') of Lemma 1 , the result is a function $f(z) \in \mathscr{F}$ with $\left|f^{\prime}(0)\right|>\left|\phi^{\prime}(0)\right|$.

Let $\Lambda$ be an arbitrary component of the boundary of $\Omega^{\prime}$.

Case 1. There exists a point $w_{0} \in \Lambda,\left|w_{0}\right|=1$. Then $\Delta_{\Lambda}=\{|w|<1\}-\Lambda$ is simply connected and $\Delta_{A} \supset \Omega^{\prime}$. As above, it follows by Lemma 1 that $\Delta_{A}=\{|w|<1\}$ and hence $\Lambda=\{|w|=1\}$.

Case 2. $\Lambda$ does not meet $\{|w|=1\}$. Then $\Lambda$ is a circular slit concentric with $|w|=1$. Otherwise, we could map the doubly connected region $\Delta_{\Lambda}\left(\supset \Omega^{\prime}\right)$ onto the unit disk minus a concentric circular slit, preserving the origin and $|w|=1$, and the result is, again by (1') a function $f \in \mathscr{F}$ with $\left|f^{\prime}(0)\right|>\left|\phi^{\prime}(0)\right|$. This proves the theorem.

3. Some properties of $\phi(z)$. To study $\phi(z)$ further we derive several additional lemmas. Let $D$ be a doubly connected circular slit region with outer boundary $|z|=1$ and slit $\sigma=\left\{z \mid z=r e^{i \theta}, \theta_{1} \leq \theta \leq \theta_{1}+\alpha\right\}$. 
Let $D^{\prime}$ be another region of this type in the $w$-plane with slit $\sigma^{\prime}=$ $\left\{w \mid w=\rho e^{i \theta}, \theta_{1}^{\prime} \leq \theta \leq \theta_{1}^{\prime}+\beta\right\}$. Let $R$ be the annulus $\{\zeta|q<| \zeta \mid<1\}$, $q>0$.

Lemma 2. Suppose $w=f(z)$ provides a one-to-one conformal map of $D$ onto $D^{\prime}$ such that $f(0)=0$ and $\sigma$ is mapped onto $|w|=1$. (Hence $\sigma^{\prime}$ will correspond to $|z|=1$.) Then

$$
\left|f^{\prime}(0)\right|=\frac{\rho}{r} .
$$

Proof. We may suppose $\sigma$ to be symmetrical with respect to the real axis. The function $F(z)=\log [(f(z) / z)(z-r)]$ is regular in $D$ once a branch of $F(0)=\log \left(-r f^{\prime}(0)\right)$ is chosen. Let $\sigma(\delta)$ be a curvilinear quadrilateral whose sides are $\{|z|=r \pm \delta,-\alpha / 2-\delta \leq \theta \leq \alpha / 2+\delta\}$ and $\{r-\delta \leq|z| \leq r+\delta, \theta= \pm(\alpha / 2+\delta)\}$. Let $C_{\delta}=\sigma(\delta) \cup\{|z|=1\}$.

By Cauchy's formula

$$
\log \left|r f^{\prime}(0)\right|=\mathscr{R}\left\{\frac{1}{2 \pi i} \int_{\sigma_{\delta}} \frac{F(z)}{z} d z\right\} .
$$

Hence

$$
\log \left|r f^{\prime}(0)\right|=\frac{1}{2 \pi} \int_{0}^{2 \pi}\left(\log \rho+\log \left|e^{i \theta}-r\right|\right) d \theta-\mathscr{R} \frac{1}{2 \pi i} \int_{\sigma_{\delta}} \frac{F(z)}{z} d z .
$$

Now

$$
\begin{aligned}
& \frac{1}{2 \pi} \int_{0}^{2 \pi} \log \left|e^{i \theta}-r\right| d \theta=\frac{1}{2 \pi} \int_{0}^{2 \pi} \log \left|\frac{e^{i \theta}-r}{1-e^{i \theta} r}\left(1-e^{i \theta} r\right)\right| d \theta \\
& \quad=\frac{1}{2 \pi} \int_{0}^{2 \pi} \log \left|1-e^{i \theta} r\right| d \theta=0 .
\end{aligned}
$$

Furthermore, as is easily seen,

$$
\mathscr{R}\left\{\frac{1}{2 \pi i} \int_{\sigma_{\delta}} \frac{F(z)}{z} d z\right\}=o(1) \text { as } \delta \rightarrow 0 \text {. }
$$

Thus $\log \left[r\left|f^{\prime}(0)\right|\right]=\log \rho$, or $\left|f^{\prime}(0)\right|=\rho / r$.

LEMma 3. Suppose $w=g(\zeta)$ provides a one-to-one conformal map of $R$ onto $D^{\prime}$ in such a way that the outer boundaries of $R$ and $D^{\prime}$ correspond. If $g\left(\zeta_{0}\right)=0$ then $\left|\zeta_{0}\right|=\rho$.

Proof. This lemma may be obtained from an explicit formula for $g(\zeta)$ as given by Komatu [10], but it may also be proved in an elementary way as follows. 
Any branch of $F(\zeta)=1 / \zeta \log g(\zeta) / \zeta-\zeta_{0}$ is regular in $R$. Hence

$$
\mathscr{R} \frac{1}{2 \pi i} \int_{\partial R} F(\zeta) d \zeta=0 \text {. }
$$

We have

$$
\begin{aligned}
-\mathscr{R}\left[\frac{1}{2 \pi i} \int_{\partial R} \frac{1}{\zeta} \log \left(\zeta-\zeta_{0}\right) d \zeta\right] & =\mathscr{R}\left\{\frac{1}{2 \pi i}\left[\int_{|\zeta|=q}-\int_{|\zeta|=1}\right]\right\} \\
& =\mathscr{R} \log \left(-\zeta_{0}\right)+\frac{1}{2 \pi} \int_{0}^{2 \pi} \log \left|e^{i \theta}-\zeta_{0}\right| d \theta \\
& =\log \left|\zeta_{0}\right|
\end{aligned}
$$

and

$$
\mathscr{R}\left\{\frac{1}{2 \pi i} \int_{\partial R} \log g(\zeta) \frac{d \zeta}{\zeta}\right\}=-\log \rho
$$

Hence, $\rho=\left|\zeta_{0}\right|$, as was to be shown.

Now let $\mu_{D}$ be the harmonic measure of $\sigma$ with respect to $D$, evaluated at the origin, i.e., if $\omega(z)$ is harmonic in $D$ with boundary values $\omega(z)=0,|z|=1$, and $\omega(z)=1, z \in \sigma$, then $\mu=\omega(0)$. Also let $q=q_{D}$ be the inner radius obtained when $D$ is mapped onto an annulus $R_{D}$. (The numbers $\mu_{D}$ and $q_{D}$ are completely determined by $r$ and $\alpha$.)

Lemma 4. Let $f(z)$ be defined as in Lemma 2. Then

$$
\left|f^{\prime}(0)\right|=q_{D}^{1-2 \mu_{D}}
$$

Hence $\left|f^{\prime}(0)\right|>1$ if and only if $\mu_{D}>1 / 2$.

Proof. Let $D$ be mapped onto $R_{D}$ in such a way that the outer boundaries of $D$ and $R_{D}$ correspond. If $\zeta=\zeta(z)$ is the mapping function.

$$
\omega(z)=\frac{\log |\zeta(z)|}{\log q_{D}} .
$$

Hence, if $\zeta_{0} \in R$ is the point corresponding to the origin in $D$, we must have

$$
\mu_{D}=\frac{\log \left|\zeta_{0}\right|}{\log q_{D}}
$$

By Lemma 3. $\left|\zeta_{0}\right|=r$. Hence $r=q_{D}^{\mu}$. Now $q_{D^{\prime}}=q_{D}$, and $\mu_{D^{\prime}}=1-\mu_{D}$ (due to the fact that the inner boundary of $D$ corresponds to the outer boundary of $D^{\prime}$ ). Therefore $\rho=q_{D}^{1-\mu} D$. The assertion now follows by Lemma 2. We may note that if $\alpha \geq \pi$ this is sufficient to guarantee 
that $\mu_{D}=1 / 2 \pi \int_{0}^{2 \pi} \omega\left(r e^{i \theta}\right) d \theta>1 / 2 \pi \int_{\theta_{1}}^{\theta_{1}+\pi} d \theta=1 / 2$. Hence $\alpha \geq \pi$ implies $\left|f^{\prime}(0)\right|>1$.

In view of Lemma 4 we can draw the following conclusion regarding the domain $\Omega^{\prime}=\phi(\Omega)$ of Theorem 1. Let the arc $\Lambda$ be an arbitrary inner component of $\partial \Omega^{\prime}$ and $\mu_{A}$ the value of the harmonic measure of $\Lambda$ with respect to $\Delta_{A}=\{|w|<1\}-\Lambda$ at the origin. Then we have

Theorem 2. For any inner boundary component $\Lambda$ of $\phi(\Omega), \mu_{\Lambda} \leq 1 / 2$. In particular all inner boundary components of $\phi(\Omega)$ subtend angles less than $\pi$ at the origin.

Proof. If a boundary component $\Lambda$ with $\mu_{\Lambda}>1 / 2$ existed, form $\phi_{1}(z)=f(\phi(z))$, where $f(z)$ maps the doubly connected region $\Delta_{A}=$ $\{|w|<1\}-\Lambda$ conformally onto a circular slit unit disk such that $f(0)=0$ and $\Lambda$ corresponds to the unit circle. Then, by Lemma $4,\left|\phi_{1}^{\prime}(0)\right|>\phi^{\prime}(0) \mid$, contrary to the definition of $\phi(z)$.

4. Mappings preserving the outer boundary component. The extremal function $\phi(z)$ of the family $\mathscr{F}$ obtained in Theorem 1 is not necessarily unique. For a region of finite connectivity $\phi(z)$ is uniquely determined if $\phi$ is subjected to the additional restriction that it carries a prescribed boundary component into the circle $|w|=1$. In this case $\phi(z)$ is also the only mapping function of $\Omega$ onto a circular slit disk with $\phi(0)=0, \phi^{\prime}(0)>0$. Lemma 4 shows for the case in which $\Omega=D$, a disk with only one slit $\sigma$, that there may exist two distinct function $\phi_{1}(z)$, $\phi_{2}(z)$ which map $D$ onto a circular slit disk and for which $\phi_{1}^{\prime}(0)=\phi_{2}^{\prime}(0)=1$. One of these carries $|z|=1$ into the slit of $\Sigma$ and the other into the outer boundary $|w|=1$. This is the case when $\mu_{D}=1 / 2$. These functions $\phi_{1}$ and $\phi_{2}$ are necessarily extremal functions of $\mathscr{F}$ in $D$.

We now prove, ${ }^{6}$ for the case of an arbitrary bounded region $\Omega$, the

${ }^{6}$ For the purpose of what follows it is convenient to use in place of the usual definition of a boundary component (viz. a boundary continuum which is not a proper subset of any connected subset of $\partial \Omega$ ) an alternate definition. Let $\left\{\Omega_{n}\right\}$ be a sequence of subregions of $\Omega$ such that (i) $\Omega_{n+1} \subset \Omega_{n}$, (ii) the relative boundary $\partial_{n} \cap \Omega$ consists of a single closed (rectifiable) Jordan curve, (iii) $\cap_{n=1}^{\infty} \Omega_{n}=0$. Two sequences $\left\{\Omega_{n}\right\},\left\{\Omega_{n}^{\prime}\right\}$ are called equivalent if, for any $n$, there exist $m$ such that $\Omega_{m} \subset \Omega_{n}^{\prime}$ and $\Omega_{m}^{\prime} \subset \Omega_{n}$. A boundary component of $\Omega$ is defined as an equivalence class of $\left\{\Omega_{n}\right\}$. Then it is known that, given any component $B$ of $\partial \Omega$, there exists a sequence $\left\{\Omega_{n}\right\}$ such that $B=\cap_{n=1}^{\infty} \bar{\Omega}_{n}$ and conversely, given a sequence $\left\{\Omega_{n}\right\}$, the set $\cap_{n=1}^{\infty} \bar{\Omega}_{n}$ is a component $B$ of $\partial \Omega$ in the usual sense.

Let $w=f(z)$ be a topological mapping of $\Omega$ onto a plane region $\Omega^{\prime}$. Then the present definition establishes a one-to-one correspondence between the boundary components of $\Omega$ and $\Omega^{\prime}$. (See Stoilow [20], pp. 85-87; Oikawa [12].) It also follows: If $\Omega$ is a region in $\mid z[\langle 1, z=0 \in \Omega$, and if $|z|=1$ is a boundary component of $\Omega$, there exists a sequence of closed Jordan curves $C_{n} \subset \Omega$, such that $C_{n}$ separates $z=0$ from $|z|=1$ and lies in the annulus $1-\varepsilon_{n}<|z|<1$ where $\lim _{n \rightarrow \infty} \varepsilon_{n}=0$. 
existence and uniqueness of the extremal function which preserves the outer boundary component.

THEOREM 3. Let $\Omega$ be a bounded region which contains $z=0$. Let $\mathscr{F}^{*}$ be the class of functions $f(z)$ with the properties:

1. $f(z)$ is analytic and univalent in $\Omega$.

2. $f(0)=0, f^{\prime}(0)>0,|f(z)| \leq 1, z \in \Omega$.

3. The outer boundary component of $\Omega$ corresponds to the outer boundary component of $f(\Omega)$.

Then there exists a function $\phi(z) \in \mathscr{F}^{*}$ for which

$$
\phi^{\prime}(0)=\max _{f \in \mathscr{F}^{*}} f^{\prime}(0)
$$

and $\phi(z)$ maps $\Omega$ onto a circular slit unit disk. $\phi(z)$ is unique.

Proof. The existence of a function $\phi(z)$ which satisfies (7) and the properties 1 and 2 of the class $\mathscr{F}^{*}$, and which maps $\Omega$ onto a circular slit unit disk is seen as in the proof of Theorem 1. The fact that $\phi(z)$ also possesses property 3 of the class $\mathscr{F}^{*}$ follows from Lemma 5 below. The uniqueness will be proved in Theorem 4 .

Lemma 5. Suppose $\left\{f_{n}(z)\right\}$ is a sequence of functions analytic and univalent in a bounded region $\Omega$, which contains $z=0$. Suppose that $f_{n}(0)=0,\left|f_{n}(z)\right| \leq 1$, and that under the mapping $w=f_{n}(z)$ the outer boundary component $B$ of $\Omega$ corresponds to the outer boundary component of $f_{n}(\Omega)$. If $\lim _{n \rightarrow \infty} f_{n}(z)=\phi(z) \not \equiv$ const. in $\Omega$, uniformly on any compact subset, then, under the mapping $w=\phi(z), B$ corresponds to the outer boundary component of $\phi(\Omega)$.

Proof. We may assume $B$ to be the circle $|z|=1$. There exists a sequence of closed rectifiable Jordan curves, $C_{k}$, such that $C_{k}$ lies in the annulus $1-\varepsilon_{k}<|z|<1, \lim _{k \rightarrow \infty} \varepsilon_{k}=0$, and such that $C_{k}$ separates $z=0$ from $|z|=1\left(\mathrm{see}^{6}\right)$. If $\Gamma_{k}^{(n)}=f_{n}\left(C_{k}\right)$, then as is easily seen $\Gamma_{k}^{(n)}$ separates $w=0$ from the outer component $B_{n}^{\prime}$ of $f_{n}(\Omega)$. Thus

$$
1=\frac{1}{2 \pi i} \int_{\Gamma_{k}^{(n)}} \frac{d w}{w}=\frac{1}{2 \pi i} \int_{\sigma_{k}} \frac{f_{n}^{\prime}(z)}{f_{n}(z)} d z
$$

In the last integral we let $n \rightarrow \infty$ and obtain, if $\phi\left(C_{k}\right)=\Gamma_{k}$,

$$
1=\frac{1}{2 \pi i} \int_{\sigma_{k}} \frac{\phi^{\prime}(z)}{\phi(z)} d z=\frac{1}{2 \pi i} \int_{r_{k}^{\prime}} \frac{d w}{w}
$$

which shows that $\Gamma_{k}=\phi\left(C_{k}\right)$ separates $w=0$ from the outer boundary component $B^{\prime}$ of $\phi(\Omega)$. 
Now, if $\Lambda$ is any component of $\partial \Omega$ different from $|z|=1$, then it is entirely contained in the interior $I\left(C_{k}\right)$ of $C_{k}$ for sufficiently large $k$. Since $z=0$ may then be connected with some point of $\Lambda$ by an arc $\gamma$ in $I\left(C_{k}\right) \cap \Omega$ (except for its endpoint on $\Lambda$ ), the boundary component $\Lambda^{\prime}$ corresponding to $\Lambda$ under the mapping $w=\phi(z)$ must lie in $I\left(\Gamma_{k}\right)$. Thus $\Gamma_{k}$ separates $\Lambda^{\prime}$ from $B^{\prime}$. Hence no component of $\partial \Omega$ except for $\{|z|=$ 1 ) can correspond to $B^{\prime}$. This proves the lemma.

5. Uniqueness of the extremal function of $\mathscr{F}^{*}$. Let $\Omega$ be a bounded region which contains $z=0$ and let $\left\{\Omega_{n}\right\}$ be an exhaustion of $\Omega$, i.e., the regions $\Omega_{n}$ have the properties:

1. $z=0 \in \Omega_{n} \subset \Omega_{n+1} \subset \Omega$.

2. $\Omega_{n}$ is bounded by $n$ closed rectifiable Jordan curves $C_{\nu}^{(n)}, \nu=$ $1,2, \cdots, n$, such that $C_{n}^{(n)}$ is the outer boundary component of $\Omega_{n}$.

3. $\bigcup_{\nu=1}^{\infty} \Omega_{\nu}=\Omega$.

Let $\mathscr{F}^{*}$ be the class of functions defined in Theorem 3 and $\phi(z)$ an extremal function of $\mathscr{F}^{*}$. Let $\mathscr{F}_{n}^{*}$ be the corresponding class for $\Omega_{n}$ and $\phi_{n}(z)$ an extremal function of $\mathscr{F}_{n}^{*}$. Let $\Sigma_{n}$ denote the circular slit disk $\phi_{n}\left(\Omega_{n}\right)$, and let $\Sigma$ be $\phi(\Omega)$.

THEOREM 4. $\lim _{n \rightarrow \infty} \phi_{n}(z)=\phi(z)$ uniformly in any compact set in S. Hence the solution of the extremal problem (7) is unique.

Proof. The functions $\left\{\phi_{n}(z)\right\}$ form a normal family in any subregion of $\Omega$. Let $\left\{\phi_{n_{p}}(z)\right\}$ be a convergent subsequence, $g(z)=\lim _{p \rightarrow \infty} \phi_{n_{p}}(z)$. If say, $\Omega \subset\{|w|<1\}$ then $\phi_{n}^{\prime}(0) \geq 1$ (the function $z$ being a member of $\mathscr{F}_{n}^{*}$.) Hence also $g^{\prime}(0) \geq 1$, so that $g(z) \not \equiv$ const. and hence univalent in $\Omega$.

We note that $g(z) \in \mathscr{F}^{*}$. Conditions 1 and 2 are clearly satisfied. To verify Condition 3 we note first: If $C$ is any closed rectifiable Jordan curve in $\Omega$ which contains $z=0$ in its interior, then $\Gamma=g(C)$ contains $w=0$ in its interior. For sufficiently large $n, C \subset \Omega_{n}$; consider the subregion of $\Omega_{n}$ which is bounded by $C$ and the contours $C_{\nu}^{(n)}$ which are contained in $I(C)$. Then

$$
1=\frac{1}{2 \pi i} \int_{\sigma} \frac{\phi_{n}^{\prime}(z)}{\phi_{n}(z)} d z-\frac{1}{2 \pi i} \sum_{\nu} \int_{C_{\nu}^{(n)}} \frac{\phi_{n}^{\prime}(z)}{\phi_{n}(z)} d z .
$$

Since $\phi_{n}\left(C_{\nu}^{(n)}\right)$ is a slit it follows that each integral of the last sum is 0 . Letting $n \rightarrow \infty$ we obtain

$$
1=\frac{1}{2 \pi i} \int_{0} \frac{g^{\prime}(z)}{g(z)} d z=\frac{1}{2 \pi i} \int_{\Gamma} \frac{d w}{w}
$$

i.e., $w=0 \in I(\Gamma)$. 
Now it is seen as in the proof of Lemma 5 that any component $\Lambda$ of $\partial \Omega$ which is different from the outer component $B$ will be in the intorior of a suitable closed Jordan curve $C$ in $\Omega$ which separates $z=0$ from $B$. If $\Lambda$ corresponds to $\Lambda^{\prime}$ then $\Lambda^{\prime}$ will be in the interior of $\Gamma=$ $g(C)$, so that $\Gamma$ separates $\Lambda^{\prime}$ from the outer boundary component of $g(\Omega)$. Since $g(z) \in \mathscr{F}^{*}$ we have

$$
g^{\prime}(0) \leq \phi^{\prime}(0) .
$$

We apply now Lemma 1 with $f_{n}(w)=\phi\left[\phi_{n}^{-1}(w)\right], F_{n}(z)=\log \left\{f_{n}(w) / w\right\} \equiv$ $\log \left\{\phi(z) / \phi_{n}(z)\right\}\left(z \in \Omega_{n}\right)$, where $F_{n}(0)=\log \phi^{\prime}(0)-\log \phi_{n}^{\prime}(0)$ is real. We find

$$
\exp \left[\frac{1}{2 \pi} \iint_{\Omega_{n}}\left|F_{n}^{\prime}(z)\right|^{2} d x d y\right] \leq \frac{\phi_{n}^{\prime}(0)}{\phi^{\prime}(0)} .
$$

Here we take $n=n_{k}$ and observe that by (8), $\lim _{k \rightarrow \infty} \phi_{n_{k}}^{\prime}(0) / \phi^{\prime}(0) \leq 1$. Let $F(z)=\log \{\phi(z) / g(z)\}, \mathfrak{J n} F(0)=0$. Then, as $k \rightarrow \infty$, we have in any closed subdomain $S \subset \Omega$,

$$
\frac{1}{2 \pi} \iint_{S}\left|F^{\prime}(z)\right|^{2} d x d y=0
$$

and therefore $g(z) \equiv \phi(z)$.

Since any convergent subsequence of $\left\{\phi_{n}\right\}$ has the limit $\phi(z)$, the whole sequence converges.

CoRollary. The domain $\Sigma$ onto which the extremal function $\phi$ of $\mathscr{T}^{*}$ maps $\Omega$ has Lebesgue area $\pi$. Hence $\partial \Sigma$ has measure 0.

Proof. By (1'), applied to $f=f_{n}(w)$ we obtain

$$
\left.\mid f_{n}^{\prime}(0)\right]^{2}=\left[\frac{\phi^{\prime}(0)}{\phi_{n}^{\prime}(0)}\right]^{2} \leq \frac{A_{n}}{\pi}=\frac{1}{\pi} \iint_{\Omega_{n}}\left|\phi^{\prime}(z)\right|^{2} d x d y .
$$

For $n=n_{k}$ we find from (8), letting $k \rightarrow \infty$,

$$
\pi \leq \lim _{k \rightarrow \infty} \int_{\Omega_{n_{k}}} \int_{\eta_{k}}\left|\phi^{\prime}(z)\right|^{2} d x d y=\iint_{\Omega}\left|\phi^{\prime}(z)\right|^{2} d x d y .
$$

Since the right side does not exceed $\pi$ the conclusion follows.

6. Extremal slit disks. It is well known that the conformal transformation of a bounded region $\Omega$ onto a circular slit disk by means of a function $f \in \mathscr{F}^{*}=\mathscr{F}^{*}(\Omega)$ need not be unique. ${ }^{7}$ To characterize a

7 For example, if $\Omega$ is a circular slit unit disk with positive (two-dimensional) slit :neasure, the identity and the extremal function for $\mathscr{F}^{*}\left(\Omega^{j}\right)$ provide two distinct mappings of class $\mathscr{F}(\Omega)$ onto circular slit disks. 
class of disks onto which there is only one (normalized) conformal map possible we introduce the following.

DEFINITION. $\Sigma$ is called an extremal circular slit unit disk (e.s.d.) if the extremal function $\psi(w)$ of $\mathscr{F}^{*}(\Sigma)$, i.e., the solution of problem (7) over $\Sigma$, is the identity, $\psi(w) \equiv w .^{8}$

Two consequences of this definition are immediate:

1. The set $S$ of the slits of an e.s.d. $\Sigma$ has two-dimensional measure 0 . This follows from the Corollary of Theorem 4, applied to the extremal function $\psi(w)$ of $\mathscr{F}^{*}(\Sigma)$.

2. If the circular projection $E$ of the slits of a circular slit disk $\Sigma$ onto a fixed radius has linear measure zero, then $\Sigma$ is an e.s.d. For in this case we can apply Lemma 1 to the extremal function $\psi(w)$ of $\mathscr{F}^{*}(\Sigma)$ and find $\psi^{\prime}(0) \leq 1$. Since the function $w \in \mathscr{F}^{*}(\Sigma)$ we have $\psi^{\prime}(0) \geq 1$ and hence $\psi^{\prime}(0)=1$ and, because of the uniqueness of the extremal function, $\psi(w) \equiv w$.

One can characterize an extremal slit disk in terms of extremal length. Suppose the annulus $A_{q}: 0<q<|z|<1$ contains all slits of a circular slit unit disk $\Sigma$. Let $S_{q}=A_{q} \cap \Sigma$ and let $\lambda_{q}\{\gamma\}$ denote the extremal length of the family of closed rectifiable curves in $S_{q}$ which separate $|z|=1$ from $|z|=q$. Denote by $\Lambda_{q}\{\gamma\}$ the extremal length of the corresponding family $\{\gamma\}$ in $A_{q}$, so that $\Lambda_{q}\{\gamma\}=2 \pi / \log (1 / q)$. Then:

THEOREM 5. A necessary and sufficient condition that $\Sigma$ be an extremal circular slit disk is that for some $q<1$, for which $A_{q}$ contains all slits of $\Sigma$,

$$
\lambda_{q}\{\gamma\}=\Lambda_{q}\{\gamma\}=\frac{2 \pi}{\log \frac{1}{q}}^{9}
$$

REMARK. If (9) holds for some $q$ it is also true for all $p, 0<p \leq q$. This is easily seen by use of elementary properties of the extremal length, since, for the family $\{\gamma\}$ in the annulus $p<|z|<q$, the extremal length $\Lambda\{\gamma\}=2 \pi / \log (q / p)$.

8 This is the analogue to Koebe's "Minimaler Schlitzbereich" [9] in the case of the parallel slit mapping. Grötzsch [5] considered the corresponding domain for the mapping on a circular slit annulus and denoted it as "Normalbereich." $\mathrm{He}$ defined the Normalbereich by means of his "Extremalprinzip" [5], p. 239 which he formulated first for the parallel slit domain in [4]. Our characterization of an e.s.d. below is suggested by Grötzsch' Extremalenprinzip.

9 Theorem 5 could also be obtained by use of inequalities due to Oikawa [12] and Jurchescu [7]. Cf. also Strebel [22], and Sakai [23], where similar, but not identical characterizations for mappings onto annuli are considered. 
Proof. Suppose $\Sigma$ is an extremal slit disk. Since $A_{q} \supset S_{q}$ we have

$$
\lambda_{q}\{\gamma\} \geq \Lambda_{q}\{\gamma\}=\frac{2 \pi}{\log \frac{1}{q}} .
$$

Let $\left\{D_{n}\right\}$ be an exhaustion of $\Sigma$, where $D_{n}$ is bounded by $n$ rectifiable closed Jordan curves. We assume that the disk $|z| \leq q$ is contained in all $D_{n}$. Let $\phi_{n}(z)$ be the extremal function in $\mathscr{F}^{*}\left(D_{n}\right)=\mathscr{F}_{n}^{*} ; \phi_{n}(z)$ maps $D_{n}$ onto a circular slit unit disk $\Sigma_{n}$ with $n-1$ slits. Let $q_{n}=$ $\max _{|z|=q}\left|\phi_{n}(z)\right|$. Since $\Sigma$ is an e.s.d., $\phi_{n}(z) \rightarrow z$, uniformly in any compact subset of $\Sigma$ (Theorem 4) and, therefore, $q_{n} \rightarrow q$ as $n \rightarrow \infty$.

Let $S_{q}^{(n)}=A_{q} \cap D_{n}$. If $\lambda_{q}\{\gamma\}_{n}$ denotes the extremal length of the family $\{\gamma\}$ of $S_{q}^{(n)}$, then

$$
\lambda_{q}\{\gamma\} \leq \lambda_{q}\{\gamma\}_{n}
$$

since $S_{q}^{(n)} \subset S_{q}$. Now $\lambda_{q}\{\gamma\}_{n}$ may be calculated in the region $\phi_{n}\left(S_{q}^{(n)}\right)$. But this region contains the annulus $q_{n}<|w|<1$ minus the slits of $\Sigma_{n}$ within this ring. If we denote the extremal length of the family $\{\gamma\}$ confined to this slit annulus by $\Lambda_{q_{n}}\{\gamma\}$ we have

$$
\lambda_{q}\{\gamma\}_{n} \leq A_{q_{n}}\{\gamma\} .
$$

From the fundamental properties of the extremal length it follows that the extremal length of the family $\{\gamma\}$ is an annulus slit along a finite number of slits is the same as that in the annulus itself. Hence

$$
\Lambda_{q_{n}}\{\gamma\}=\frac{2 \pi}{\log \frac{1}{q_{n}}} .
$$

Thus we obtain

$$
\frac{2 \pi}{\log \frac{1}{q}} \leq \lambda_{q}\{\gamma\} \leq \frac{2 \pi}{\log \frac{1}{q_{n}}}
$$

and letting $n \rightarrow \infty$ we find (9).

Suppose now, conversely, that (9) is satisfied. Let $\phi(z)$ be the extremal function in $\mathscr{F}^{*}(\Sigma)$ and choose $\rho=\left|\phi^{\prime}(z) / \phi(z)\right|$ as a metric in $S_{q}=A_{q} \cap \Sigma$. Then, from the definition of extremal length,

$$
\lambda_{q}\{\gamma\} \geq \frac{(2 \pi)^{2}}{\iint\left|\frac{\phi^{\prime}(z)}{\phi(z)}\right|^{2} d x d y} \geq \frac{(2 \pi)^{2}}{\iint_{S_{q}} \frac{d u d v}{|w|^{2}}}
$$


where $q^{\prime}=\min _{|z|=q}|\phi(z)| . \quad$ Since $\iint_{q^{\prime}<|w|<1} d u d v /|w|^{2}=\int_{0}^{2 \pi} \int_{q^{\prime}}^{1} 1 / r d r d \theta=$ $2 \pi \log 1 / q^{\prime}$ we obtain

$$
\lambda_{q}\{\gamma\} \geq \frac{2 \pi}{\log \frac{1}{q^{\prime}}}=\frac{2 \pi}{\log \frac{1}{q}+\log \frac{q}{q^{\prime}}} .
$$

Suppose that $\Sigma$ were not an e.s.d. Then $\phi^{\prime}(0)>1$. Since (by the Remark above) we may assume $q$ arbitrarily small, we note that $\lim _{q \rightarrow 0} \log q / q^{\prime}=\log 1 / \phi^{\prime}(0)<0$, and hence, for a suitable $\delta>0$,

$$
\lambda_{q}\{\gamma\} \geq \frac{2 \pi}{\log \frac{1}{q}-\delta}>\frac{2 \pi}{\log \frac{1}{q}},
$$

which contradicts the present hypothesis.

Theorem 6. Let $\Sigma$ be a circular slit unit disk. Then the conclusion (1) of Lemma 1 holds for all $f \in \mathscr{F}^{*}(\Sigma)$ if and only if $\Sigma$ is an extremal circular slit disk.

Proof. (a) Suppose $\Sigma$ is an e.s.d. Consider an exhaustion $\left\{D_{n}\right\}$ of $\Sigma$, where $D_{n}$ is bounded by $n$ closed rectifiable Jordan curves; $z=0 \cong D_{n}$. Let $\zeta=\phi_{n}(z)$ be the extremal function of $\mathscr{F}_{n}^{*}=\mathscr{F}^{*}\left(D_{n}\right) ; \phi_{n}(z)$ maps $D_{n}$ conformally onto a circular slit disk $\Sigma_{n}$ with $n-1$ slits. We apply Lemma 1 to $f_{n}(\zeta)=f\left[\phi_{n}^{-1}(\zeta)\right]$ and

$$
F_{n}(z)=\log \frac{f_{n}(\zeta)}{\zeta}=\log \frac{f(z)}{\phi_{n}(z)}
$$

where $F_{n}(0)=\log f^{\prime}(0)-\log \phi_{n}^{\prime}(0)$ and $\log \phi_{n}^{\prime}(0)$ is real. Let $A_{n}=$ $\iint_{D_{n}}\left|f^{\prime}(z)\right|^{2} d x d y ; A_{n}$ is the area of $f\left(D_{n}\right)$. Then we obtain

$$
\exp \left[\frac{1}{2 \pi} \iint_{D_{n}}\left|F_{n}^{\prime}(z)\right|^{2} d x d y\right] \cdot \frac{\left|f^{\prime}(0)\right|}{\phi_{n}^{\prime}(0)} \leq \sqrt{\frac{A_{n}}{\pi}}
$$

Since $\Sigma$ is an e.s.d., we have by Theorem $4, \lim _{n \rightarrow \infty} \phi_{n}(z)=z$, uniformly in any compact subset of $\Sigma$. Furthermore, using the lower semicontinuity of the Dirichlet integral on the left and the fact that $\lim _{n \rightarrow \infty} A_{n}=A$, the area of $f(\Sigma)$, on the right, we obtain with $F(z)=\log f(z) / z$,

$$
\exp \left[\frac{1}{2 \pi} \iint_{\Sigma}\left|F^{\prime}(z)\right|^{2} d x d y\right]\left|f^{\prime}(0)\right| \leq \sqrt{\frac{A}{\pi}} .
$$

(b) Suppose conversely, that (1) is true for every $f \in \mathscr{F}^{*}(\Sigma)$. Then 
we apply (1) to the extremal function $\phi$ in this class and find

$$
\phi^{\prime}(0) \leq 1 \text {. }
$$

Since the function $f=z \in \mathscr{F}^{*}(\Sigma)$, we have $\phi^{\prime}(0) \geq 1$, hence $\phi^{\prime}(0)=1$, and, therefore, in view of the uniqueness of the extremal function, $\phi(z) \equiv z$.

THEOREM 7. Let $\Omega$ be a bounded region which contains $z=0$. There is at most one conformal map, $f \in \mathscr{F}(\Omega)$, of $\Omega$ onto an extremal circular slit unit disk which carries a given component of $\partial \Omega$ into $|w|=1$.

Proof. Suppose there were two such mappings, $f_{1}$ and $f_{2}, f_{1}(\Omega)=$ $\Sigma_{1}, f_{2}(\Omega)=\Sigma_{2}$. Then $g=f_{1} \circ f_{2}^{-1} \in \mathscr{F}^{*}\left(\Sigma_{2}\right)$.

Since $\Sigma_{2}$ is an e.s.d. we have, by Theorem $6, g^{\prime}(0) \leq 1$. The same argument applies to $f_{2} \circ f_{1}^{-1}$ and shows that $g^{\prime}(0) \geq 1$. Hence, $g^{\prime}(0)=1$ and $g(w) \equiv w$ or $f_{1} \equiv f_{2}$.

THEOREM 8. If $\phi$ is an extremal function in $\mathscr{T}(\Omega)$, then $\phi(\Omega)=\Sigma$ is an extremal slit disk.

Proof. Let $\psi(w)$ denote the extremal function of $\mathscr{F}^{*}(\Sigma)$. If $\Sigma$ were not an e.s.d., then we would have $\psi^{\prime}(0)>1$. Then $f=\psi(\phi(z)) \in \mathscr{F}(\Omega)$, but $f^{\prime}(0)=\psi^{\prime}(0) \phi^{\prime}(0)>\phi^{\prime}(0)$, which contradicts the definition of $\phi$.

REMARK. In view of Theorem 2 it is clear that the class of all possible domains $\phi(\Omega)$, produced by an extremal function $\phi$ of $\mathscr{F}(\Omega)$ is a proper subclass of the class of all extremal slit disks.

7. Mapping onto a radial slit disk. Suppose $\Omega$ is a bounded region which has a free boundary component $B$, not a single isolated point. We may assume that $B$ is the circle $|z|=1$ and that $\Omega$ is contained in $|z|<1$ and contains an annulus $\rho<|z|<1$. We suppose also that $z=0 \in \Omega$.

Let $(5)$ denote the class of functions $g(z)$ with the properties:

1. $g(z)$ is analytic and univalent in $\Omega$ and on $|z|=1$.

2. $g(0)=0, g^{\prime}(0)>0$ and $|g(z)| \leq 1, z \in \Omega$.

3. $|g(z)|=1$ for $|z|=1$.

THeOREM 9. There exists a unique function $\psi(z) \in(B)$ such that $\psi^{\prime}(0)=\min _{g \in \mathbb{B}} g^{\prime}(0) ; \psi(z)$ maps $\Omega$ conformally onto a radial slit disk of radius one such that $|z|=1$ corresponds to $|w|=1 .^{9}$

${ }^{9}$ See Sario [16] where this mapping is obtained by a different extremal problem. An example by Oikawa [12] which is modeled after a construction of Strebel [21] indicates that the mapping of an arbitrary bounded region onto a radial slit disk is not always possible, 
We shall need the following lemma which plays a similar role in the proof of this theorem as Lemma 1 in the case of the circular slit disk mapping.

Lemma 6 . Let $\Sigma$ be a radial slit unit disk centered at $z=0$ such that the closure of the radial projection of the slits onto $|z|=1$ has measure 0. Suppose $w=f(z)$ is analytic and univalent in $\Sigma$, that $f(0)=0$ and that $f(z)$ maps $\Sigma$ onto a bounded region $\Delta$ in such a manner that $|z|=1$ corresponds to the outer boundary component of 4. Let $m=\inf _{|z| \rightarrow 1}|f(z)|$, and let $A$ denote the area of $\Delta$. If $F(z)=$ $\log (f(z) / z), F(0)=\log f^{\prime}(0)$, then

$$
\exp \left[\frac{1}{2 \pi} \iint_{\bar{\Sigma}}\left|F^{\prime}(z)\right|^{2} d x d y\right] \cdot m^{2} \leq\left|f^{\prime}(0)\right| \sqrt{\frac{A}{\pi}} .
$$

In particular,

$$
m^{2} \leq\left|f^{\prime}(0)\right| \sqrt{\frac{A}{\pi}}
$$

and the equality sign implies that $F^{\prime}(z) \equiv 0$, i.e., $f(z) \equiv a z$, where $a$ is a constant.

REMARK. If $\Sigma$ has only a finite number of slits and $|f(z)| \leq M$, $z \in \Sigma$, then (10) implies Rengel's inequality $\left|f^{\prime}(0)\right| \geq m^{2} \mid M[13]$. Again, equality holds only if $f(z) \equiv a z$.

Proof. Let $E$ denote closure of the radial projection of the slits onto $|z|=1$. $E$ is a closed set of measure zero. Consequently, for every $\varepsilon>0$-and we assume $\varepsilon<m$-there exist a finite number of arcs $\left.\beta_{\nu}=\{z\}|z|=1, \theta_{\nu} \leq \theta \leq \theta_{\nu}^{\prime}\right\}$, where $\theta_{\nu}<\theta_{\nu}^{\prime}<\theta_{\nu+1}, \nu=1,2, \cdots, n$, and $\theta_{n+1}=\theta_{1}+2 \pi$, such that

$$
E \subset \bigcup_{\nu=1}^{n} \beta_{\nu} \text { and } \sum_{\nu=1}^{n}\left(\theta_{\nu}^{\prime}-\theta_{\nu}\right)<\varepsilon .
$$

We may assume that $\theta_{\nu} \notin E, \theta_{\nu}^{\prime} \notin E$. Let $|z| \leq d$ be a disk contained in $\Sigma$ and let $\delta>0(1-\delta>d)$ be so small that, for $1-\delta \leq|z|<1$, $z \in \Sigma,|f(z)| \geqq m-\varepsilon$.

We denote by $c_{\nu}$ the arc composed of the following three parts: the two segments $\left\{z\left|\theta=\theta_{\nu}, d \leq\right| z \mid \leq 1-\delta\right\},\left\{z\left|\theta=\theta_{\nu}^{\prime}, d \leq\right| z \mid \leq 1-\delta\right\}$ and the arc $\left\{z|| z \mid=d, \theta_{\nu} \leq \theta \leq \theta_{\nu}^{\prime}\right\}$. Let $\gamma_{\nu}=\left\{z|| z \mid=1-\delta, \theta_{\nu}^{\prime} \leq \theta \leq\right.$ $\left.\theta_{\nu+1}\right\}, \nu=1,2, \cdots, n$. Then we define $C_{\delta}=\left(\bigcup_{\nu=1}^{n} c_{\nu}\right) \cup\left(\bigcup_{\nu=1}^{n} \gamma_{\nu}\right) ; C_{\delta}$ is a closed Jordan curve in $\Sigma$ which contains the origin in its interior $D_{\delta}$, and $F(z)$ is regular in $D_{\delta} \cup C_{\delta}$. We assume again at first that $f^{\prime}(0)=1, F(0)=0$. We write $F(z)=u+i v, f(z)=\rho e^{i, r}$ and obtain 


$$
\iint_{D_{\delta}}\left|F^{\prime}(z)\right|^{2} d x d y=\int_{\sigma_{\delta}} u d v=\int_{\sigma_{\delta}} \log \frac{\rho}{r}(d \psi-d \theta) .
$$

Using the fact that $F(0)=0$ and consequently

$$
\Im n 1\left\{\int_{c_{\delta}} \frac{F(z)}{z} d z\right\}=\int_{c_{\delta}}(\log r d \psi-\log \rho d \theta)=0
$$

we find

$$
\iint_{D_{\delta}}\left|F^{\prime}(z)\right|^{2} d x d y=\int_{C_{\delta}}(\log \rho d \psi-2 \log \rho d \theta+\log r d \theta) .
$$

Since $\int_{c_{\delta}} \log r d \theta \leq 0$, we have

$$
\iint_{D_{\delta}}\left|F^{\prime}(z)\right|^{2} d x d y+2 \int_{c_{\delta}} \log \rho d \theta \leq \int_{c_{\delta}} \log \rho d \psi
$$

Let $\rho_{0}=\min _{|z|=l}|f(z)|$. Writing $\theta_{\nu}^{\prime}-\theta_{\nu}=\left|\beta_{\nu}\right|$ and using (12) we obtain

$$
\begin{aligned}
\int_{\sigma_{\delta}} \log \rho d \theta & \geq \log \rho_{0} \sum_{\nu=1}^{n}\left|\beta_{\nu}\right|+\log (m-\varepsilon)\left[2 \pi-\sum_{\nu=1}^{n}\left|\beta_{\nu}\right|\right] \\
& \geq 2 \pi \log (m-\varepsilon)-\lambda \varepsilon
\end{aligned}
$$

where $\lambda$ is a positive constant.

Let $\Gamma_{\delta}=f\left(C_{\delta}\right), \Delta_{\delta}=f\left(D_{\delta}\right), k$ the disk $|w| \leq \mu$, where $\mu$ is so small that $k \subset \Delta_{\delta}$. We have

$$
\int_{C_{\S}} \log \rho d \psi=\int_{\Gamma_{\delta}} \log \rho d \psi=\iint_{\lrcorner_{\delta}-k} \frac{d u d v}{|w|^{2}}+2 \pi \log \mu .
$$

Just as in the proof of Lemma 1 we then obtain

$$
\int_{\sigma_{\delta}} \log \rho d \psi \leq 2 \pi \log \sqrt{\frac{A}{\pi}} .
$$

Thus we have from (13), (14), and (15)

$$
\iint_{D_{\delta}}\left|F^{\prime}(z)\right|^{2} d x d y+4 \pi \log (m-\varepsilon) \leq 2 \pi \log \sqrt{\frac{A}{\pi}}+2 \lambda \varepsilon .
$$

Letting first $\delta \rightarrow 0$ and then observing that the resulting inequality holds for every $\varepsilon, 0<\varepsilon<m$, we obtain (10) when $f^{\prime}(0)=1$.

If $f^{\prime}(0) \neq 1$, let $a=\left|f^{\prime}(0)\right|$. Then we need only to replace $f(z)$ by $f(z) / a, A$ by $A / a^{2}$ and $m$ by $m / a$ to obtain (10).

Proof of Theorem 9. Since (S) is a normal family in $\Omega$ there exists a sequence $\left\{g_{n}(z)\right\}, g_{n} \in(B)$, which converges to a function $\psi(z)$ and for 
which $\lim _{n \rightarrow \infty} g_{n}^{\prime}(0)=\operatorname{Inf}_{g \in \mathbb{S}} g^{\prime}(0)$. Clearly, $\psi(z)$ is analytic in $\Omega, \psi(0)=$ $0,|\psi(z)| \leq 1$. We show that $\psi(z) \not \equiv$ constant. Let $P_{\rho}$ be the annulus $\rho<|z|<1$ contained in $\Omega$. Then for every $r, \rho<r<1,\left|g_{n}\left(r e^{i \theta}\right)\right|$ assumes the value $r$. Otherwise the image of $|z|=r$ lies entirely either in the interior or in the exterior of $|z|=r$. In either case the ring onto which $w=g_{n}(z)$ maps $P_{r}: r<|z|<1$ cannot have the module $\log (1 / r)$ (which it must have). Because of the uniform convergence of $\left\{g_{n}(z)\right\}$ on $|z|=r,\left|\psi\left(r e^{i \theta}\right)\right|$ assume the value $r$ somewhere on $|z|=r$. Hence $\psi \not \equiv$ const. Consequently $\psi$ is univalent in $\Omega, \psi^{\prime}(0)>0$, and $\psi(z) \neq 0$ in $P_{\rho}$ since $\psi(0)=0$.

Each $g \in$ (S) may be continued analytically across $|z|=1$ by the relation

$$
g(z)=\frac{1}{\bar{g}\left(\frac{1}{\bar{z}}\right)} \quad\left(1<|z|<\frac{1}{o}\right)
$$

If $\rho<r<1$, the fact that $g_{n}(z)$ converges uniformly to $\psi(z)$ on $|z|=r$ and that $\psi(z) \neq 0$ implies that, uniformly on $|z|=1 / r, \lim _{n \rightarrow \infty} g_{n}(z)=$ $1 / \bar{\psi}(1 / \bar{z}) \equiv \psi(z)$.

The uniform convergence of $g_{n}$ for $|z|=r$ and $|z|=1 / r$ implies that $g_{n}(z) \rightarrow \psi(z)$ uniformly in the ring $r<|z|<1 / r$ and therefore in particular on $|z|=1$; hence $|\psi(z)|=1$ for $|z|=1$.

The function $\psi(z)$ maps, therefore, $\Omega$ onto a region $\Sigma$ in $|w|<1$ and $|z|=1$ onto $|w|=1$. We maintain that all other boundary components of $\Sigma$ which are not points must be radial slits. For, if $A$ is a boundary component of $\Sigma$ in $|w|<1$ which is not a slit, we map the doubly connected region $\Delta$ consisting of the complement of $\Lambda$ with respect to $|w|<1$ onto a radial slit unit disk. By Lemma 6 the mapping function $\zeta=h(w)$ has the property $\left|h^{\prime}(0)\right|<1$; hence $h[\psi(z)] \in \mathbb{B}$ and $\left|h^{\prime}(0) \psi^{\prime}(0)\right|<\left|\psi^{\prime}(0)\right|$ contrary to the construction of $\psi(z)$.

The uniqueness of $\psi(z)$ follows from Theorem 10 below.

8. Uniqueness of the extremal function of 18 . Suppose $\left\{\Omega_{n}\right\}$ denotes an exhaustion of the region $\Omega$ considered in $\S 7$, of the following type:

1. $z=0 \in \Omega_{n} \subset \Omega_{n+1} \subset \Omega$.

2. Each $\Omega_{n}$ is bounded by the circle $|z|=1$ and $n-1$ closed Jordan curves $C_{\nu}^{(n)}, \nu=1,2, \cdots, n-1$, contained in $|z|<1$ and exterior each other.

3. $\Omega=\bigcup_{n=1}^{\infty} \Omega_{n}$.

It follows, in particular, that the annulus $P_{\rho}: \rho<|z|<1$, which is in $\Omega$, is also contained in all $\Omega_{n}$ for sufficiently large $n$.

Let $\mathscr{S}_{n}$ denote the family $\left(S\right.$ defined in $\Omega_{n}$ and let $\psi_{n}(z)$ be an extremal function in $\mathscr{S}_{n}$, such that $\psi_{n}^{\prime}(0)=\min _{g \in \aleph_{n}} g^{\prime}(0) ; \psi_{n}(z)$ maps $\Omega_{n}$ 
onto a radial slit disk $\Sigma_{n}$ having $n-1$ slits. Let $\psi(z)$ be the function defined in Theorem 9 and let $\Sigma=\psi(\Omega)$.

THEOREM 10. Uniformly in any compact subset of $\Omega, \lim _{n \rightarrow \infty} \psi_{n}(z)=$ $\psi(z)$. Hence the extremal function of Theorem 9 is unique.

Proof. Since the functions $\left\{\psi_{n}(z)\right\}$ form a normal family in any fixed $\Omega_{m}(n \geq m)$, there exists a convergent subsequence $\left\{\psi_{n_{k}}\right\}$. Let $\gamma(z)=$ $\lim _{k \rightarrow \infty} \psi_{n_{k}}(z)$. Just as in the proof of Theorem 9, by applying the argument to a fixed region $\Omega_{m}$ in place of $\Omega$, one shows that $\gamma(z)$ is analytic on the unit circle and that $|\gamma(z)|=1$ for $|z|=1$. Thus $\gamma(z) \in \mathbb{B}$, and we have, therefore,

$$
\gamma^{\prime}(0) \geq \psi^{\prime}(0)
$$

We apply now Lemma 6 to $f_{n}(w)=\psi\left(\psi_{n}^{-1}(w)\right)$,

$$
F_{n}(z)=\log \frac{\psi\left(\psi_{n}^{-1}(w)\right)}{w} \equiv \log \frac{\psi(z)}{\psi_{n}(z)}, \quad z \in \Omega_{n},
$$

where $F_{n}(0)=\log \psi^{\prime}(0) \cdot \log \psi_{n}^{\prime}(0)$ is real. Noting that $m=1, A \leq \pi$, we obtain

$$
\left.\exp \left[\frac{1}{2 \pi}\right] \iint_{\Omega_{n}}\left|F_{n}^{\prime}(z)\right|^{2} d x d y\right] \leq \frac{\psi^{\prime}(0)}{\psi_{n}^{\prime}(0)}
$$

Let $F(z)=\log \{\psi(z) / \gamma(z)\}$ where $F(0)=\log \left\{\psi^{\prime}(0) / \gamma^{\prime}(0)\right\}$ is real. Then we find for every compact subset $S \subset \Omega$, as $k \rightarrow \infty$, because of (16),

$$
\frac{1}{2 \pi} \iint_{S}\left|F^{\prime}(z)\right|^{2} d x d y=0 \text {. }
$$

Hence $F^{\prime}(z) \equiv 0$ and consequently $\psi(z) \equiv \gamma(z)$.

Since every convergent subsequence of $\left\{\psi_{n}\right\}$ has the limit $\psi$ the conclusion of the theorem follows.

CoRollary. The Lebesgue area of the domain $\Sigma$ onto which the extremal function $\psi(z)$ of $(\mathrm{S}$ maps $\Omega$ is $\pi$. Hence the measure of $\partial \Sigma$ is zero.

The proof is entirely analogous to that of the corollary to Theorem 4 .

9. Extremal radial slit disks. Let $\Sigma$ be a radial slit unit disk such that $|z|=1$ is an isolated boundary component. $\Sigma$ shall be called an extremal radial slit unit disk (e.r.s.d.) if the extremal function $\psi$ of $\mathbb{S}(\Sigma)$ is the identity, $\psi(w) \equiv w$.

Clearly, the slit measure of an e.r.s.d. is zero. Furthermore, if the closure of the radial projection of the slits of a radial slit unit disk $\Sigma$ 
has (linear) measure 0 , then $\Sigma$ is an e.r.s.d.

An e.r.s.d. may be characterized in terms of the extremal length of a suitable family $\{\gamma\}$. Let $\Sigma$ be a radial slit unit disk with isolated outer boundary, let $A_{q}: q<|z|<1$ be an annulus containing all slits of $\Sigma$, and let $S_{q}=A_{q} \cap \Sigma$. Suppose $\lambda_{q}^{*}\{\gamma\}$ denotes the extremal length of all rectifiable curves $\gamma$ in $S_{q}$ (except for the endpoints) which join $|z|=q$ to $|z|=1$. Let $\Lambda_{q}^{*}\{\gamma\}$ be the extremal length of the corresponding family $\{\gamma\}$ in $A_{q}$.

THEOREm 11. Necessary and sufficient that $\Sigma$ be an e.r.s.d. is that $\lambda_{q}^{*}\{\gamma\}=\Lambda_{q}^{*}\{\gamma\}=1 / 2 \pi \log 1 / q$, for some $q$, for which $A_{q}$ contains all slits of $\Sigma$.

The proof is entirely analogous to that of Theorem $5 .^{10}$

Finally, one extends the validity of Lemma 6 to an extremal radial slit disk in a manner parallel to the extension of Lemma 1 to Theorem 6 , and one proves by use of this extension:

THEOREM 12. If $g \in \mathbb{B}=\mathbb{S}(\Omega)$ and if $\Sigma=g(\Omega)$ is an extremal radial slit unit disk, then $g$ is the extremal function $\psi \in \mathbb{B}$.

\section{BIBLIOGRAPHY}

1. L. V. Ahlfors and L. Sario, Riemann Surfaces, Princeton University Press, 1960.

2. G. M. Golusin, Geometrische Funktionentheorie (translation from the Russian) VEB Deutscher Verlag der Wissenschaften, Berlin, 1957.

3. H. Grötzsch, Über konforme Abbildung unendlich vielfach zusammenhängender schlichter Bereiche mit endlich vielen Häufungsrandkomponenten, Sächsische Akad. der Wissenschaften, Berichte, 81 (1929), 51-86.

4. - Zum Parallelschlitatheorem der konformen Abbildung schlichter unendlich vielfach zusammenhängender Bereiche, ibid., 83 (1931) 185-200.

5. — Das Kreisbogenschlitztheorem der konformen Abbildung schlichter Bereiche, ibid., 83 (1931), 238-253.

6. H. Grunsky, Neue Abschätzungen zur konformen Abbildung ein-und mehrfach zusammenhängender Bereiche, Schriften des Math. Seminars und des Instituts f. angewandte Math. der University Berlin, 1 (1932), 95-140.

7. M. Jurchescu, Modulus of a boundary component, Pacific J. Math., 8 (1958), 791-809.

8. P. Koebe, Abhandlungen zur Theorie der konformen Abbildung. IV. Abbildung mehrfach zusammenhängender schlichter Bereiche auf Schlitzbereiche, Acta Math., 41 (1918), 305-344.

9. —, Zur konformen Abbildung unendlich vielfach zusammenhängender schlichter Bereiche auf Schlitzbereiche, Göttinger Nachrichten, (1918), 60-71.

10. Y. Komatu, Zur konformen Abbildung zweifach zusammenhängender Gebiete, I, II, Proceedings of the Japan Academy, 21 (1945), 285-307.

11. Z. Nehari, Conformal Mapping, McGraw-Hill, New York, 1952.

12. K. Oikawa, On the stability of boundary components, doctoral dissertation, University of California, Los Angeles, (1958), 1-128, Pacific J. Math., 10 (1960), 263-294.

10 In the proof one makes use of the fact that $\Lambda_{q}^{*}\{\gamma\}$ remains unchanged if one removes from $A_{q}$ a finite number of radial slits. This statement was proved by Strebel [21], p. 5 . 
13. E. Rengel, Über einige Schlitztheoreme der konformen Abbildung, Schriften des Mathematischen Seminars und der Instituts für angewandte Mathematik, der Univ. Berlin 1 (1932), 141-162.

14. - Existenzbeweise für schlichte Abbildungen mehrfach zusammenhängender Bereiche auf gewisse Normalbereiche, Jahresbericht d. Deutschen Math., 44 (1934), 51-7.

15. L. Sario, Capacity of the boundary and of a boundary component, Annals of Mathematics, 59 (1954), 135-144.

16. - Strong and weak boundary components, J. d'analyse math. (Jerusalem), 5 (1956/57), 389-398.

17. M. Schiffer, Sur un théorème de la représentation conforme, C. R. Acad. Sci., Paris, 207 (1938), 520-522.

18. - Some recent developments in the theory of conformal mapping, Appendix to R. Courant, Dirichlets Principle, Conformal Mapping, and Minimal Surfaces, Interscience Publishers, (1950), 249-323.

19. D. C. Spencer, Distortion in conformal mapping, supplement to S. Bergman, Partial differential equations, mimeographed notes, Brown University, 1941.

20. S. Stoilow, Leçons sur les Principes Topologiques de la Théorie des Fonctions Analytiques, Gauthier-Villars, Paris, 1938.

21. K. Strebel, Eine Ungleichung für extremale Längen, Annales Acad. Scient. Fennicae A. I 90 (1951), 1-7.

22. - A remark on the extremal distance of two boundary components, Procs. Nat. Acad. Sci., 40 (1954), 842-844.

23. A. Sakai, On minimal slit domains, Procs. Japan Academy, 35 (1959), 128-133.

The University of Minnesota and

UNIVERSity OF CALIFornia, LOS ANGElES 



\section{PACIFIC JOURNAL OF MATHEMATICS}

\section{EDITORS}

\author{
David Gilbarg \\ Stanford University \\ Stanford, California \\ F. H. Brownell \\ University of Washington \\ Seattle 5 , Washington
}

\author{
A. L. Whiteman \\ University of Southern California \\ Los Angeles 7, California \\ L. J. PAIGE \\ University of California \\ Los Angeles 24, California
}

\section{ASSOCIATE EDITORS}

\author{
E. F. BECKENBACH \\ T. M. CHERRY \\ D. DERRY
}
E. HEWITT
A. HORN
L. NACHBIN
M. OHTSUKA
H. L. ROYDEN
M. M. SCHIFFER

E. SPANIER

E. G. STRAUS

F. WOLF

\section{SUPPORTING INSTITUTIONS}

\author{
UNIVERSITY OF BRITISH COLUMBIA \\ CALIFORNIA INSTITUTE OF TECHNOLOGY \\ UNIVERSITY OF CALIFORNIA \\ MONTANA STATE UNIVERSITY \\ UNIVERSITY OF NEVADA \\ NEW MEXICO STATE UNIVERSITY \\ OREGON STATE COLLEGE \\ UNIVERSITY OF OREGON \\ OSAKA UNIVERSITY \\ UNIVERSITY OF SOUTHERN CALIFORNIA
}

\author{
STANFORD UNIVERSITY \\ UNIVERSITY OF TOKYO \\ UNIVERSITY OF UTAH \\ WASHINGTON STATE COLLEGE \\ UNIVERSITY OF WASHINGTON

AMERICAN MATHEMATICAL SOCIETY
CALIFORNIA RESEARCH CORPORATION
HUGHES AIRCRAFT COMPANY
SPACE TECHNOLOGY LABORATORIES
NAVAL ORDNANCE TEST STATION

Mathematical papers intended for publication in the Pacific Journal of Mathematics should be typewritten (double spaced), and the author should keep a complete copy. Manuscripts may be sent to any one of the four editors. All other communications to the editors should be addressed to the managing editor, L. J. Paige at the University of California, Los Angeles 24, California. ..

50 reprints per author of each article are furnished free of charge; additional copies may be obtained at cost in multiples of 50 .

The Pacific Journal of Mathematics is published quarterly, in March, June, September, and December. The price per volume (4 numbers) is $\$ 12.00$; single issues, $\$ 3.50$. Back numbers are available. Special price to individual faculty members of supporting institutions and to individual members of the American Mathematical Society: $\$ 4.00$ per volume; single issues, $\$ 1.25$.

Subscriptions, orders for back numbers, and changes of address should be sent to Pacific Journal of Mathematics, 2120 Oxford Street, Berkeley 4, California.

Printed at Kokusai Bunken Insatsusha (International Academic Printing Co., Ltd.), No. $6_{4}$ 2-chome, Fujimi-cho, Chiyoda-ku, Tokyo, Japan.

PUBLISHED BY PACIFIC JOURNAL OF MATHEMATICS, A NON-PROFIT CORPORATION

The Supporting Institutions listed above contribute to the cost of publication of this Journăl, but they are not owners or publishers and have no responsibility for its content or policies. 


\section{Pacific Journal of Mathematics}

\section{Vol. 10, No. $3 \quad$ November, 1960}

Glen Earl Baxter, An analytic problem whose solution follows from a simple

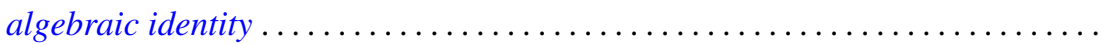

Leonard D. Berkovitz and Melvin Dresher, A multimove infinite game with linear payoff. .

Earl Robert Berkson, Sequel to a paper of A. E. Taylor ......................

Gerald Berman and Robert Jerome Silverman, Embedding of algebraic systems.... 767

Peter Crawley, Lattices whose congruences form a boolean algebra . . . . . ...... 777

Robert E. Edwards, Integral bases in inductive limit spaces . . . . . . . . . . . . . . .

Daniel T. Finkbeiner, II, Irreducible congruence relations on lattices . . . . . . . . . .

William James Firey, Isoperimetric ratios of Reuleaux polygons . . . . . . . . . . . 787

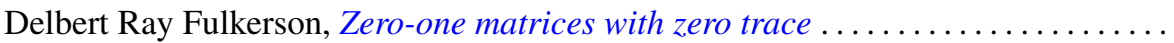

Leon W. Green, A sphere characterization related to Blaschke's conjecture........

Israel (Yitzchak) Nathan Herstein and Erwin Kleinfeld, Lie mappings in

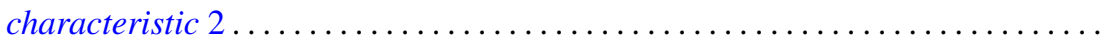

Charles Ray Hobby, A characteristic subgroup of a $p$-group .................

R. K. Juberg, On the Dirichlet problem for certain higher order parabolic

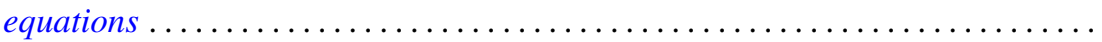

Melvin Katz, Infinitely repeatable games ......................

Emma Lehmer, On Jacobi functions . . . . . . . . . . . . . . . . . . . . . . . . .

D. H. Lehmer, Power character matrices

Henry B. Mann, A refinement of the fundamental theorem on the density of the sum of two sets of integers.

Marvin David Marcus and Roy Westwick, Linear maps on skew symmetric matrices: the invariance of elementary symmetric functions . .

Richard Dean Mayer and Richard Scott Pierce, Boolean algebras with ordered

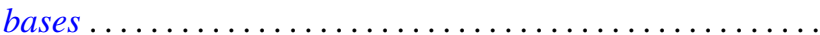

Trevor James McMinn, On the line segments of a convex surface in $E_{3} \ldots$

Frank Albert Raymond, The end point compactification of manifolds ..

Edgar Reich and S. E. Warschawski, On canonical conformal maps of regions of arbitrary connectivity

Marvin Rosenblum, The absolute continuity of Toeplitz's matrices...

Lee Albert Rubel, Maximal means and Tauberian theorems . .

Helmut Heinrich Schaefer, Some spectral properties of positive linear operators

Jeremiah Milton Stark, Minimum problems in the theory of pseudo-conformal transformations and their application to estimation of the curvature of the invariant metric.

Robert Steinberg, The simplicity of certain groups ...

Hisahiro Tamano, On paracompactness. .

Angus E. Taylor, Mittag-Leffler expansions and spectral theory .

Marion Franklin Tinsley, Permanents of cyclic matrices ...... . 